\title{
Decisional Balance Inventory (DBI) Adolescent Form for Smoking: Psychometric Properties of the Persian Version
}

Maryam Khazaee-Pool ${ }^{1 *}$ (D, Tahereh Pashaei ${ }^{2,7}$, Koen Ponnet $^{3,4}$, Fatemeh Jafari $^{5}$ and Rashin Alizadeh ${ }^{6}$

\begin{abstract}
Background: One effective model for studying cigarette smoking cessation is the transtheoretical model (TTM). In order to assess to what degree interventions can make variations in individuals' behavior, several questionnaires have been developed based on the TTM. This study aims to describe the development of the Persian version of the Decisional Balance Inventory (DBI) for smoking cessation in Iran and to evaluate its psychometric properties.

Design and methods: The forward-backward technique was used to translate the DBI from English into Persian. After linguistic validation and a pilot test among 30 male smoking young adults, a cross-sectional study was performed, and psychometric properties of the Persian version of the DBI were assessed. Using a convenience sampling method, 120 male smokers between 16 and 24 years of age were recruited from three factories in Nowshahr, Iran. In order to assess the reliability of the DBI, internal consistency and test-retest methods were performed. Additionally, face and content validity were assessed, and the construct validity of the DBI was calculated by performing both exploratory and confirmatory factor analysis. Data were analyzed using SPSS and AMOS.
\end{abstract}

Results: The mean age of the sample $(n=120)$ was 20.19 ( $S D=2.13)$ years. The mean scores for the content validity index (CVI) and the content validity ratio (CVR) were .94 and .89, respectively. The results of exploratory factor analysis (EFA) showed a three-factor solution for the DBI that accounted for $55.4 \%$ of observed variance. The results achieved from the confirmatory factor analysis (CFA) displayed that the data fit the model: the relative chi-square $(\times 2 / \mathrm{df})=1.733$ $(p<.001)$ and the root mean square error of approximation (RMSEA $)=.07(90 \% \mathrm{Cl}=.05-.105)$. All comparative indices of the model including GFI, AGFI, CFI, NNFI, and NFI were more than $.80(.87, .83, .91, .89$, and .81 , respectively). The Cronbach's alpha ranged from .78 to .83 , indicating an acceptable reliability. Furthermore, the intraclass correlation coefficient (ICC) ranged from .72 to .89 , confirming a satisfactory result.

Conclusions: The results from the present study indicate that the Persian version of the DBI has good psychometric properties and is suitable to measure smoking behaviors among Iranian adolescent and young adult smokers. Consequently, the instrument could be used in planning cigarette smoking cessation interventions among Iranian adolescents and young adults.

Keywords: Decisional balance inventory, Reliability, Validity, Smokers

\footnotetext{
*Correspondence: khazaee.m@zums.ac.ir; khazaie_m@yahoo.com

'Department of Health Education and Promotion, School of Public Health,

Zanjan University of Medical Sciences, Zanjan, Iran

Full list of author information is available at the end of the article
}

(c) The Author(s). 2017 Open Access This article is distributed under the terms of the Creative Commons Attribution 4.0 International License (http://creativecommons.org/licenses/by/4.0/), which permits unrestricted use, distribution, and reproduction in any medium, provided you give appropriate credit to the original author(s) and the source, provide a link to the Creative Commons license, and indicate if changes were made. The Creative Commons Public Domain Dedication waiver (http://creativecommons.org/publicdomain/zero/1.0/) applies to the data made available in this article, unless otherwise stated. 


\section{Background}

One preventable cause of early mortality is cigarette smoking [1]. Although the tobacco smoking rate is reducing overall, more than two-thirds of recent deaths in developing countries are caused by diseases related to smoking tobacco. Based on a report of the World Health Organization (WHO), 22\% of the world's population aged 15 years and above are smokers, and almost 6 million people die from exposure to tobacco smoke or from tobacco use [2]. According to a study from 2012, the prevalence rate of current daily smoking in Iran is respectively $11.3 \%$ (21.4\% of men and $1.4 \%$ of women) and $12.5 \%$ (23.4\% of men and $1.4 \%$ of women). Furthermore, it was reported that the mean number of cigarettes smoked every day by Iranian smokers was 13.7 sticks [1].

The large number of population still use cigarette smoking in spite of its risky impacts. It is recognized that quitting cigarette smoking results in several health benefits, such as reduced mortality risk due to cardiovascular diseases $[3,4]$. Because of the strongly addictive nature of nicotine, relapse after stopping is common. Furthermore, there are some social benefits of smoking that prevent smokers from quitting, like enhanced feelings of relaxation and a sense of control [5-7]. Persons who smoke for a long time are commonly not influenced by the long-term benefits of quitting cigarette smoking, especially when the diseases that are associated with smoking have not developed obviously [8-10].

Interpersonal communication has an important effect on smoking. From the perspective of social cognition, the decision of whether or not to smoke is influenced by the response of peers in one's social environment. For instance, individuals may be discouraged from smoking cigarettes if they believe that smoking is perceived as a negative behavior by the public and that they may face social disapproval by doing so. As such, the negative social consequences to smoking, such as peer refusal, are closely associated with a decreased probability of continued smoking [11, 12]. Thus, individuals who received negative consequences may have less of a tendency to smoke cigarettes [13].

Successful approaches to the cessation of cigarette smoking are often based on behavioral change models [14]. The transtheoretical model (TTM) is known as one of the most important models in the field of preventive health behavior $[15,16]$. This model, developed by Prochaska, assesses an individual's readiness to act on a new, healthier behavior and provides approaches for change to guide the individual [17]. The TTM is composed of four constructs: stages of change, processes of change, self-efficacy, and decisional balance and temptations [18-20]. The TTM is based on the assumption that people are at different stages of motivational readiness for engaging in healthier behaviors and that intervention approaches are most effective when they are matched to a person's current stage of change [15, 21]. Decisional balance, which is the focus of the present article, refers to the idea that pros and cons are important in the decision-making process for behavioral change.

Based on the TTM, several scales were developed to assess to what degree interventions can cause variations in individuals' behavior. One of the TTM-based scales that measure lifestyle changes like smoking is the Decisional Balance Inventory (DBI), which measures positive thoughts (pros) and negative thoughts (cons) that might occur to an individual who is deciding whether or not to smoke [22]. The initial scale comprised 24 items, but later on, Pallonen et al. (1998) developed a DBI with 12 items [23]. In developed countries, the English version of the DBI has been validated in many studies [24-27], but unfortunately, there is limited literature on this topic in developing countries like Iran .

There is need for a questionnaire on health behavior changes that can be applied in various cultural settings. Culturally and linguistically competent scales like the DBI consider cultural values, beliefs, and practices that vary among diverse people. So, it is essential to re-assess the validity and reliability of this scale in a specific culture, like the Iranian one. Therefore, the aim of this study is to examine the psychometric properties of the Persian version of the DBI for smoking cessation in Iranian adolescents and young adults. Consistent with other studies, albeit in a different context [24-27], we expect the Persian version of the DBI to have good psychometric properties.

\section{Methods}

\section{The Decisional Balance Inventory (DBI)}

The DBI is a self-report instrument that focuses on either a positive thought (pro) or a negative thought (con) that might happen to a person who is deciding whether or not to smoke or not smoke. The original DBI was developed by Velicer and comprises 24 items that assess the opinions of adolescents about the damages and benefits of smoking [22]. The brief DBI was developed by Pallonen in 1998 and consists of 12 items. The shortened DBI measures one of the key constructs of the TTM and consists of three factors, including cons of smoking (six items), social pros (three items) and coping pros (three items), and each item is rated on a five-point Likert-type scale $(1=n o t$ important to 5 = extremely important) [23].

\section{Translation}

After getting permission from the author, the forwardbackward method was used to translate the DBI from English into Persian. For the forward translation, two independent expert translators translated the scale into Persian. Then, the Persian versions were compared by one of the authors and both of the translators, and they 
made a single temporary Persian version of the DBI. For the backward step, two other English professionals translated the temporary Persian version of the DBI back into English, and a temporary English version was made. Both translators were fluent in both Persian and English, and they were also skilled health care experts who have been employed for many years. In the next step, the study team and the translators tested the scale for accuracy. In order to measure the content validity of the DBI, an expert panel including seven health professionals (two psychologists, two health education experts, one epidemiologist, and two specialists in tobacco control) compared the provisional English version of the DBI with the original scale, and after some verbal and cultural adaptations, the pre-final Persian version of the DBI was made. This version was assessed in a pilot study with 30 male smoking adolescents. Eventually, the final Persian version of the DBI was produced, and it was used in this study [28].

\section{Design and data collection}

A cross-sectional validation study was carried out in Nowshahr, Iran. A convenient sample of male smokers between 16 and 24 years of age who worked in three factories in Nowshahr, Mazandaran participated in the study. The inclusions criteria were (a) being a current smoker who has smoked at least 100 cigarettes total, (b) not having participated in any effort to quit, (c) planning to quit smoking in the next 30 days, and (d) having the ability to read and write in Farsi. We recruited individuals who were in this stage to quit smoking because we were planning to perform an intervention for such a group. According to the TTM, every stage of change needs its own approach to ensure a successful intervention [18]. Therefore, we believed that the best suited sample for this study would be a sample of participants who were in a preparation stage to quit smoking. Otherwise, we would have had to create a number of different interventions for the individuals in various stages of change, that would have been difficult due to limited time and resources.

The sample size was estimated a priori. The sample size was determined based on the number of items in the scale multiplied by $10(12 \times 10=120)$ [29]. A convenience sampling method was applied to recruit the respondents. An introductory letter was sent to four factories through personal contacts of the researchers. A positive reply to cooperate on this study was received from three factories. Formal consent from the factories' manager and participants was required prior to the study. We asked the managers of the selected factories for permission to perform a study in their factory. The self-administered paper-and-pencil questionnaire was conducted during work time in the presence of a researcher, who explained the purpose and procedure of the study. Participants were assured their answers were anonymous and confidential, and that they could leave the study at any given time that they want. Afterwards, participants informed consent was achieved. The questionnaire took 25-30 min to complete.

\section{Statistical analysis}

Psychometric properties of the Persian version of the DBI were measured by the following statistical analyses:

\section{Validity}

Content, face and construct validity of the Persian version of the DBI were measured.

\section{Content validity}

Both qualitative and quantitative methods were used to assess content validity. In the qualitative stage, an expert panel consisting of seven health experts, including two psychologists, two health education experts, one epidemiologist, and two specialists in tobacco control, assessed the content validity. The experts assessed wording, grammar, item allocation and scaling of the DBI. The content validity index (CVI) and the content validity ratio (CVR) were calculated in the quantitative stage. CVI measures the relevancy, clarity, and simplicity of each item [30, 31]. In order to calculate the CVI, a Likert-type ordinal scale with four possible responses was applied. The answers were rated from $1=$ not relevant, not simple and not clear to $4=$ very relevant, very simple and very clear. The CVI was assessed as the proportion of items on a scale that attained a rating of 3 or 4 by the experts [32]. The CVR tested the essentiality of each item in a scale. In order to assess the CVR, the experts rated each item as $1=$ essential, $2=$ useful but not essential, or $3=$ not essential. Then, based on the Lawshe Table, items with a CVR score of 0.62 or above were considered to be acceptable and were retained [33].

\section{Face validity}

To assess the face validity, both qualitative and quantitative methods were used. A group of smoking male young adults $(n=10)$ were asked to evaluate each item of the scale and to indicate if they felt difficulty or ambiguity in replying to the Persian version of the DBI. Thereafter, the impact score (frequency $\times$ importance) was assessed to show the percentage of smoker men who identified each item as important or quite important on a fivepoint Likert scale. Items were considered to be appropriate if they had an impact score equal to or more than 1.5 (which corresponds to a mean frequency of $50 \%$ and a mean importance of three on the five-point Likert scale) [34]. 


\section{Construct validity}

Confirmatory factor analysis (CFA) was applied in order to assess the coherence between the data and the structure. The model fit was evaluated using multiple fit indices. As suggested, various fit indices measuring relative Chi-square, Goodness of Fit Index (GFI), Comparative Fit Index (CFI), Root Mean Square Error of Approximation (RMSEA), Non-Normed Fit Index (NNFI), Normed Fit Index (NFI) and Standardized Root Mean Square Residual (SRMR) were taken into account [35, 36]. The GFI, CFI, NFI, and NNFI value range between 0 and 1 [37], but values equal to .80 or above are commonly indicated as acceptable model fits [37]. An RMSEA value between .08 and .10 demonstrates an average fit, and a value below .08 shows a good fit. Values below .05 indicate a good fit for SRMR, but values between .05 and .08 , and between .08 and .10 indicate a close fit or are acceptable, respectively [38].

\section{Reliability}

In order to assess the reliability of the DBI, the internal consistency was tested applying the Cronbach's alpha coefficient. The alpha values equal to .70 or higher were considered acceptable [30]. Furthermore, intraclass correlation coefficient (ICC) was estimated for assessing the stability of DBI. The scale was re-administered to 40 smokers below 25 years of age 1 week after the first completion. ICC values of .40 or above are considered acceptable ( $r$ 's between .81 and 1.0 are excellent, between 0.61 and .80 are very good, between .41 and .60 are good, between .21 and .40 are fair, and between .0 and .20 are poor) [29]. The analyses were performed using the statistical program SPSS for Windows version 23.0 and Amos 24.0.

\section{Results}

\section{The study sample}

A total of 142 male smokers between 16 and 24 years of age completed the DBI. We excluded 22 questionnaires because they did not provide complete demographic information, resulting in 120 participants for analyses. The mean age of the participants was 20.19 years $(\mathrm{SD}=2.13)$. About 27\% (32 participants) had primary education, 62\% (75 participants) had secondary education, and 11\% (13 participants) had higher education. The most common age to start smoking was $13-15$ years (50.8\% participants). About $30 \%$ (36 participants) said that they started smoking between 16 and 20 years of age, and 13.3\% (16 participants) started smoking before 12 years of age [see Table 1].

\section{Validity}

An EFA was applied on the 12 items of the DBI (cutoff point: .50). Factor loadings of each item and the three subscales are presented in Table 2. All items
Table 1 Descriptive characteristics of the study sample $(n=120)$

\begin{tabular}{lll}
\hline & Number & $\%$ \\
\hline Age (years) & 26 & 21.7 \\
$\leq 18$ & 56 & 46.7 \\
$19-21$ & 38 & 31.6 \\
$22-24$ & $20.19(2.13)$ & \\
Mean (SD) & $16-24$ & \\
Range & & 85.0 \\
Marital status & 102 & 15.0 \\
Single/divorced & 18 & \\
Married & & 26.7 \\
Educational Level & 32 & 62.5 \\
Primary & 75 & 10.8 \\
Secondary & 13 & \\
Higher & 16.3 \\
Age of beginning to smoke cigarettes & 50.8 \\
$\leq 12$ & 16 & 30.0 \\
13-15 & 61 & 5.8 \\
$16-20$ & 36 & \\
$20-24$ & 7 & \\
\hline
\end{tabular}

loaded on their respective construct. The three constructs of the DBI jointly accounted for $55.4 \%$ of the observed variance.

We conducted a CFA on the 12-item questionnaire to test the fitness of the model obtained from the EFA. Fig. 1 shows the best model fit. Covariance matrixes were used and fit indexes were calculated. All fit indices proved to be good. The relative chi-square ( $\mathrm{x} 2 / \mathrm{df})$ was equal to $1.733(p<.001)$. The RMSEA of the model was .07 (90\% CI $=.05-.105)$, and the SRMR was .07. All comparative indices of the model, including GFI, AGFI, CFI, NNFI, and NFI, were more than $.80(.87, .83, .91, .89$, and .81 , respectively).

Although the model fitted good, modification indices for the regression weights were examined for identifying covariance among the factors. No significant improvement on fit indexes was obtained, so no changes were made and the model was accepted in its present form. Fig. 1 shows the model.

\section{Reliability}

To measure the internal consistency, the Cronbach's alpha was calculated separately for the DBI as well as for each factor of the DBI. The Cronbach's alpha coefficient for the DBI was .92 and ranged from .78 to .83 for its subscales, which is well above the acceptable threshold. Furthermore, test-retest analysis was conducted to test the stability of the DBI. The results indicated satisfactory results. Intra-class correlation (ICC) was .93 for the DBI 
Table 2 Exploratory factory analysis of the DBI $(n=120)$

\begin{tabular}{llll}
\hline Item & Factor 1 & Factor 2 & Factor 3 \\
\hline 6. Smoking can affect the health of others &. $\mathbf{7 5 3}$ & .041 & .146 \\
9. Smoking cigarettes is hazardous to people's health & .735 & -.012 & -.234 \\
3. Smoking stinks & .676 & .269 & .176 \\
11. Smoking is a messy habit & .659 & .074 & .249 \\
10. Cigarette smoking bothers other people & .617 & .274 \\
12. Smoking makes teeth yellow & .596 & .890 \\
1. Smoking makes kids get more respect from others & .182 & .022 \\
4. Kids who smoke have more friends & .145 &. $\mathbf{8 8 7}$ & .080 \\
7. Kids who smoke go out on more dates & .080 & .605 & .055 \\
5. Smoking cigarettes is pleasurable & .000 & -.039 \\
2. Smoking helps people to cope better with frustrations & .233 & .171 \\
8. Smoking cigarettes relieves tension & .018 & .064 \\
\hline
\end{tabular}

Note. Figures in bold relate to factor loadings equal to or higher than .50

and ranged from .72 to .89 (good to excellent) for the subscales of the DBI, lending support for the stability of the questionnaire. The results are presented in Table 3.

\section{Discussion}

The aim of the current study was to perform a psychometric evaluation for the translated Persian version of an additional component of the TTM, the Decisional Inventory Index (DBI) for smoking cessation. This scale measures movement through the stages of change and delivers insight into mechanisms through which individuals try to change their risky behaviors. In general, the results demonstrated that the translated DBI is a suitable and valid questionnaire that can be used for assessing smoking behavior among adolescent and young adult smokers who speak Persian. Developing theory-based questionnaires can be considered a main precondition for the evaluation of any intervention program.

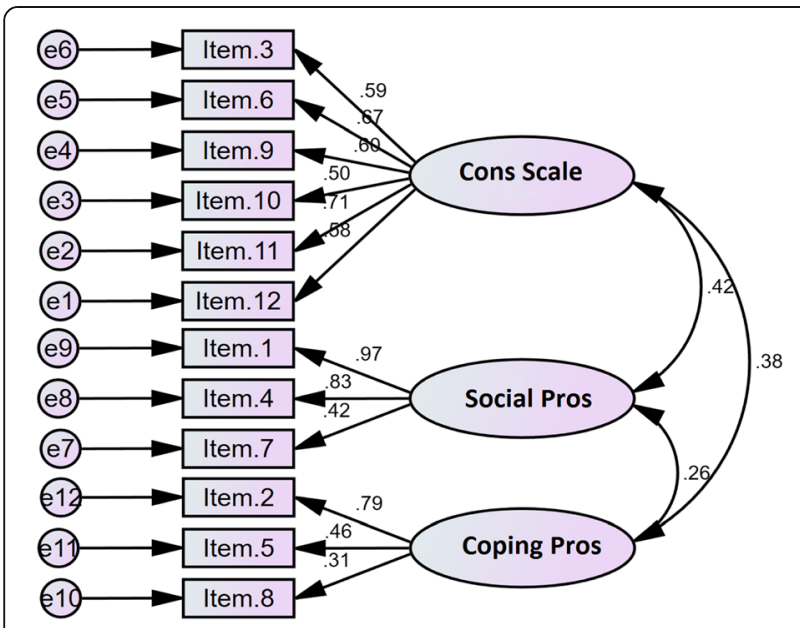

Fig. 1 A three-factor model for the DBI obtained from CFA $(n=120)$
Therefore, we consider the results from this study to be useful for adolescent and young adult who are part of a cigarette smoking control plan.

The Cronbach's alpha and the ICC were acceptable and showed good reliability and stability for the DBI. Furthermore, the CVI and the CVR showed a reasonable content validity. The EFA results were consistent with those found by the original developer of the DBI. This indicates that the DBI is effective for presenting multiple aspects of the health concerns affected by smoking. These findings are also similar to studies that have been conducted by other investigators in other contexts $[39,40]$.

As expected, the present study showed a three-factor solution for the Persian version of the DBI, including social pros as well as coping pros and cons. The three factors were able to predict $55.4 \%$ of the observed variance. This result is somewhat higher than that found by Velicier (1985), in whose study a two-factor solution (i.e., pros and cons) accounted for $41 \%$ of the observed variance [22], and it is also higher than that found by Pallonen et al. (1998), in whose study a three-factor solution (i.e., social pros, coping pros, and cons) accounted for $50 \%$ of the total variance [23]. In another study among both smokers and nonsmokers by Hoeppner et al. (2012), a four-factor solution (i.e., two pro factors, two con factors) was obtained that explained $45 \%$ of the variance [41].

The results also demonstrated that the questionnaire is able to discriminate between the perceived benefits and barriers involved in making the decision to quit smoking. Decisional balance is a key construct of the TTM, and the results of the DBI imply that pros and cons are comparatively significant parts of the model. With regard to changing health and risk behaviors among target groups, it is important to emphasize the pros and cons for that specific behavior. 
Table 3 Measures of the internal consistency and intraclass coefficient

\begin{tabular}{lllll}
\hline Factor & The name of factor & Number of items & Cronbach's alpha & ICC \\
\hline 1 & Cons Scale & 6 items $(3,11,12,9,10,6)$ & 0.800 & 0.896 \\
2 & Social Pros & 3 items $(1,4,7)$ & 0.787 & 0.724 \\
3 & Coping Pros & 3 items $(5,2,8)$ & 0.832 & 0.813 \\
Total & & 12 items & 0.928 & 0.933 \\
\hline
\end{tabular}

We also performed the CFA to determine if there was coherence between the data and the theoretical structure. The CFA provided good fit indices for the present model, and the convergent validity of the subscales of the DBI was acceptable. These findings are consistent with studies conducted in different cultural backgrounds $[22,23,42]$, which have indicated that the DBI is reliable when it is applied in Persian-speaking smokers. Our findings also demonstrated that the model from the original scale is similar to our model [23]. Furthermore, the internal consistency of the scale as measured by the Cronbach's alpha revealed an acceptable reliability for all subscales, which was consistent with previous studies $[22,23]$. Furthermore, after examining 40 male young adult smokers over a one-week period, our findings clearly indicated that the DBI has good stability in the short term; however, it has yet to be seen whether the DBI is still stable in the long term.

\section{Limitations}

The present study has also some limitations. One limitation has to do with the accuracy of the participants' answers, because all measures were self-reported. Another limitation of the current study is related to its generalizability and its sample size. The present sample was limited to a convenient sample of 120 male adolescent and young adult smokers, and it is unknown whether we would achieve the same outcomes if a large representative group of both male and female smokers were recruited. As such, the current study is unable to assess gender differences with regard to the psychometric properties of the DBI. Future studies should aim to include both male and female smokers in order to assess whether motivations for quitting smoking are similar between the genders and whether gender influences amenability to treatment. Furthermore, this study included only adolescents and young adults who were working in a factory, thus excluding students. Future studies should also assess the psychometric properties of the Persian version of the DBI in an Iranian adolescent and young adult student sample. Finally, the sample of the current study was ethnically homogenous (just Farsi); further studies need to consider the relationship between different Iranian ethnicities (e.g., Gilak, Turkish, Kurdish, Baluchi, and so on) and the DBI.

\section{Conclusion}

The findings suggest that the Persian version of the DBI is a reliable and valid scale to determine smoking behaviors among Iranian male smokers. As such, the Persian version of DBI may be supportive for healthcare teams to identify and to design health approaches that are practical and targeted to specific situations. Further studies in male and female populations are recommended to establish stronger psychometric properties for the DBI.

\section{Abbreviations \\ CFA: Confirmatory factor analysis; CFI: Comparative fit index; Cons: Negative thoughts; CVI: Content validity index; CVR: Content validity ratio; DBI: Decisional blance inventory; EFA: Exploratory factor analysis; \\ GFI: Goodness of fit index; ICC: Intraclass correlation coefficient:; NFI: Normed fit index; NNFI: Non-Normed fit index; Pros: Positive thoughts; RMSEA: Root mean square error of approximation; SRMR: Standardized root mean square residual; TTM: Transtheoretical model}

\section{Acknowledgments}

We would like to thank all participants for their contributions to this study, as well as the experts without whose support this study would not have been finished. The authors are also thankful for help received from the Department of Health Education \& Promotion, School of Health, Tehran University of Medical Sciences (TUMS).

\section{Funding}

No external funding sources were provided for this study.

\section{Availability of data and materials}

The datasets generated and analyzed during the current study are not publicly available due to protect the participants' anonymity but are available from the corresponding author on reasonable request.

\section{Authors' contributions}

MK was the main investigator, designed the project, collected the data, did the statistical analysis, and wrote the first draft of the manuscript. FJ, TP and RA contributed to the statistics. KP contributed substantially to the interpretation of the data and critically revised the final article for important intellectual content. All authors read and accepted the final paper, and agreed to be accountable for all aspects of the work in ensuring that questions related to the accuracy or integrity of any part of the work are appropriately investigated and resolved. All authors have read and approved the final version of the manuscript.

\section{Competing interests}

The authors declare that they have no competing interests.

\section{Consent for publication}

Not applicable.

\section{Ethics approval and consent to participate}

The study procedure was approved by the Medical Ethics Committee of Tehran University of Medical Sciences. This study involved only participants who gave their informed consent. For this, verbal informed consent was obtained from all participants before beginning the study, and all participants completed informed written consent after being informed about 
the purposes of the project. The questionnaire was treated as confidential and anonymous; there was no personal information that could link the responses with any of the participants in the study. To protect the rights and interests of all participants, no records of consent by name were maintained. Each completed questionnaire was returned to the researcher on the same day of data collection.

\section{Publisher's Note}

Springer Nature remains neutral with regard to jurisdictional claims in published maps and institutional affiliations.

\section{Author details}

${ }^{1}$ Department of Health Education and Promotion, School of Public Health, Zanjan University of Medical Sciences, Zanjan, Iran. ${ }^{2}$ Social Determinants of Health Research Center, Kurdistan University of Medical Sciences, Sanandaj, Iran. ${ }^{3}$ Department of Communication Sciences, Ghent University, Ghent, Belgium. ${ }^{4}$ Department of Communication Sciences, University of Antwerp, Antwerp, Belgium. ${ }^{5}$ Department of Public Health, School of Public Health, Zanjan University of Medical Sciences, Zanjan, Iran. ${ }^{6}$ Department of Health Education and Promotion, School of Health, Tehran University of Medical Sciences, Tehran, Iran. ${ }^{7}$ Department of Public Health, Faculty of Health, Kurdistan University of Medical Sciences, Sanandaj, Iran.

Received: 4 January 2017 Accepted: 15 May 2017

Published online: 25 May 2017

\section{References}

1. Meysamie A, Ghaletaki R, Zhand N, Abbasi M. Cigarette smoking in iran. Iran J Public Health. 2012;41(2):1-14.

2. Ahmadi J, Khalili H, Jooybar R, Namazi N, Mohammadagaei P. Prevalence of cigarette smoking in Iran. Psychol Rep. 2001;89(2):339-41.

3. Panagiotakos DB, Pitsavos C, Chrysohoou C, Stefanadis C, Toutouzas P. Risk stratification of coronary heart disease in Greece: final results from the CARDIO2000 Epidemiological Study. Prev Med. 2002;35(6):548-56.

4. Tavani A, Bertuzzi M, Gallus S, Negri E, La Vecchia C. Risk factors for non-fatal acute myocardial infarction in Italian women. Prev Med. 2004;39(1):128-34.

5. Hine DW, Honan CA, Marks AD, Brettschneider K. Development and validation of the Smoking Expectancy Scale for Adolescents. Psychol Assess. 2007;19(3):347-55.

6. McChargue DE, Spring B, Cook JW, Neumann CA. Reinforcement expectations explain the relationship between depressive history and smoking status in college students. Addict Behav. 2004;29(5):991-4.

7. Patterson F, Lerman C, Kaufmann VG, Neuner GA, Audrain-McGovern J. Cigarette smoking practices among American college students: review and future directions. J Am Coll Heal. 2004;52(5):203-10.

8. Aveyard P, Markham WA, Lancashire E, Almond J, Griffiths R, Cheng KK. Is inter-school variation in smoking uptake and cessation due to differences in pupil composition? A cohort study. Health Place. 2005:11(1):55-65.

9. Ellickson PL, McGuigan KA, Klein DJ. Predictors of late-onset smoking and cessation over 10 years. J Adolesc Health. 2001:29(2):101-8.

10. McDonald P, Colwell B, Backinger CL, Husten C, Maule CO. Better practices for youth tobacco cessation: evidence of review panel. Am J Health Behav. 2003;27(Suppl 2):S144-58.

11. Chassin L, Presson CC, Sherman SJ, Edwards DA. Four pathways to young-adult smoking status: adolescent social-psychological antecedents in a midwestern community sample. Health Psychol. 1991;10(6):409-18.

12. Shore $\mathrm{TH}$, Tashchian A, Adams JS. Development and validation of a scale measuring attitudes toward smoking. J Soc Psychol. 2000;140(5):615-23.

13. Agaku IT, Maliselo T, Ayo-Yusuf OA. The relationship between secondhand smoke exposure, pro-tobacco social influences, and smoking susceptibility among nonsmoking Zambian adolescents. Subst Use Misuse. 2015;50(3):387-93.

14. khazaee-Pool M, Pashaei T, Mansorian M, Qorbani M, Safari O, Shojaeizadeh D. School-Based Smoking Prevention Programs for Middle School Students in Nowshahr-Iran: a Randomized Controlled Trial. Int J Pediatr. 2016;4(11):3859-74.

15. Redding CA, Prochaska JO, Pallonen UE, Rossi JS, Velicer WF, Rossi SR. Transtheoretical individualized multimedia expert systems targeting adolescents' health behaviors. Cogn Behav Pract. 1999:6(2):144-53.

16. Prochaska JO, Velicer WF, Rossi JS, Goldstein MG, Marcus BH, Rakowski W, et al. Stages of change and decisional balance for 12 problem behaviors. Health Psychol. 1994;13(1):39-46.
17. Prochaska JO, Velicer WF. The transtheoretical model of health behavior change. Am J Health Promot. 1997;12(1):38-48.

18. Glanz K, Rimer BK, Viswanath K. Health behavior and health education: theory, research, and practice. 5th ed. Jossey-Bass: Wiley; 2008.

19. Butler JT. Principles of health education and health promotion. 3rd ed. Englewood CO: Morton; 2001.

20. Sharma M. Theoretical Foundations of Health Education and Health Promotion. 3nd Edition. Jones \& Bartlett Learning Publishers; 2016.

21. Velicer WF, Prochaska JO, Bellis JM, DiClemente CC, Rossi JS, Fava JL, et al. An expert system intervention for smoking cessation. Addict Behav. 1993:18(3):269-90

22. Velicer WF, DiClemente CC, Prochaska JO, Brandenburg N. Decisional balance measure for assessing and predicting smoking status. J Pers Soc Psychol. 1985;48(5):1279-89.

23. Pallonen UE, Prochaska JO, Velicer WF, Prokhorov AV, Smith NF. Stages of acquisition and cessation for adolescent smoking: an empirical integration. Addict Behav. 1998;23(3):303-24.

24. Hoeppner BB, Redding CA, Rossi JS, Pallonen UE, Prochaska JO, Velicer WF. Factor structure of decisional balance and temptations scales for smoking: cross-validation in urban female African-American adolescents. Int J Behav Med. 2012;19(2):217-27.

25. Guo B, Aveyard P, Fielding A, Sutton SR. The factor structure and factorial invariance for the Decisional Balance Scale for adolescent smoking. Int $J$ Behav Med. 2009:16:158-63.

26. Chen HS, Sheu JJ, Percy MS, Brown EJ, Yang RJ. The chinese version of the decisional balance scale: further validation. Nurs Res. 2006:55(4):225-30.

27. Guo B, Aveyard P, Fielding A, Sutton S. Testing the convergent and discriminant validity of the Decisional Balance Scale of the Transtheoretical Model using the Multi-Trait Multi-Method approach. Psychol Addict Behav. 2008;22(2):288-94

28. Waltz CF, Strickland OL, Lenz ER. Measurement in nursing and health research. 4rd ed. Springer Publishing Company; 2010.

29. Plichta SB, Kelvin EA, Munro BH. Statistical methods for health care research Wolters Kluwer Health/Lippincott Williams \& Wilkins Publisher; 2012

30. Cronbach $\sqcup$. Coefficient alpha and the internal structure of tests. psychometrika. 1951;16(3):297-334

31. Lynn MR. Determination and quantification of content validity. Nurs Res. 1986;35(6):382-5.

32. Waltz CF, Bausell BR. Nursing research: design statistics and computer analysis. 2nd ed. Philadelphia: FA Davis Company; 1981.

33. Lawshe $\mathrm{CH}$. A quantitative approach to content validity. Pers Psychol. 1975:28(4):563-75

34. Lacasse Y, Godbout C, Series F. Health-related quality of life in obstructive sleep apnoea. Eur Respir J. 2002:19(3):499-503.

35. Harrington D. Confirmatory factor analysis. 2nd ed. New York: Oxford University Press; 2009

36. Gable RK, Wolf MB. Instrument development in the affective domain. 2nd ed. Boston: Kluwer Academic Publishers; 1993.

37. Kline RB. Principles and practice of structural equation modeling. 3rd ed. Guilford publications; 2015.

38. Schumacker RE, Lomax RG. A beginner's guide to structural equation modeling. 3rd ed. New York, NY: Routledge; 2010.

39. Buckley TC, Kamholz BW, Mozley SL, Gulliver SB, Holohan DR, Helstrom AW, et al. A psychometric evaluation of the Smoking Consequences Questionnaire-Adult in smokers with psychiatric conditions. Nicotine Tob Res. 2005;7(5):739-45.

40. Copeland AL, Brandon TH, Quinn EP. The Smoking Consequences Questionnaire - Adult: Measurement of smoking outcome expectancies of experienced smokers. Psychol Assess. 1995:7(4):484-94.

41. Hoeppner BB, Redding CA, Rossi JS, Pallonen UE, Prochaska JO, Velicer WF. Factor structure of decisional balance and temptations scales for smoking: cross-validation in urban female African-American adolescents. Int J Behav Med. 2012;(2):19, 217-227.

42. Anatchkova MD, Redding CA, Rossi JS. Development and validation of transtheoretical model measures for Bulgarian adolescent non-smokers. Subst Use Misuse. 2007:42(1):23-41. 